\title{
Narrative Reflections on the Role of Psychiatry Residents in the Early Days of the COVID-19 Pandemic
}

\author{
Gregory E. Alberto ${ }^{1,2}$ - Brian J. Rosen ${ }^{1,2} \cdot$ Patrick A. Ho ${ }^{1,2}$ (D \\ Received: 24 March 2020 / Accepted: 12 July 2020 / Published online: 22 July 2020 \\ (C) Academic Psychiatry 2020
}

Resource scarcity within healthcare systems has been central to planning efforts as cases of COVID-19 rise in the USA. In rural areas, shortages of human resources may be especially of concern. More than any other resource shortage, we suspect personnel shortages will most significantly overwhelm the healthcare apparatus and be the most difficult to overcome. In these uncertain times, collegiality and innovative responses to the threat of COVID-19 are essential. Psychiatry residents will find themselves to be instrumental in efforts to continue providing quality care for all patients. As an example, when a high proportion of a rural academic medical center's internal medicine (IM) residents were quarantined due to COVID-19 exposure, psychiatry interns were called upon to cover their service. Here, we present the reflections and experiences of two psychiatry interns and a senior resident who becameunexpectedly - a large part of our medical center's response to this burgeoning crisis.

\section{Brian Rosen's Reflections}

I found myself missing internal medicine after beginning a 6month psychiatry rotation. I yearned for the quick fixes of diuretics or the ability to visualize gross anatomic dysfunction. Psychiatry, however, was slower paced and amorphous with a vastly different clinical skillset. Despite radically different clinical contexts, I believe that internal medicine skills were necessary for effective psychiatric practice. My internal messaging would be validated much more quickly than I could have imagined.

On March 1, I received an email from our hospital confirming that the first case of COVID-19 in our state was

\section{Patrick A. Ho}

Patrick.a.ho@hitchcock.org

1 Geisel School of Medicine at Dartmouth, Lebanon, NH, USA

2 Dartmouth-Hitchcock Residency Program, Lebanon, NH, USA an active employee. I could not have fathomed the drastic impact it would effect on my life and hospital operations. The next day I was alerted by my fiancée, an IM intern, that she and 40+ of her co-residents would be quarantined immediately. Suddenly, our largest department was without a vital clinical workforce. The $20+$ remaining residents were spread thinly across clinical sites. Almost instantaneously, rumors began to spread that interns from other services would be reassigned. It took only 4 days for rumors to become reality as psychiatry interns were reassigned to cover IM.

Despite not having thought about ward medicine for months, clinical knowledge quickly reemerged. The response from medicine to our "cross-coverage" was heartwarming. Tired peers and attendings accepted us with excitement. Over the course of my week of cross-coverage, I found myself not only filling the roll of my quarantined IM peers but also that of a psychiatric consultant. Despite our differing backgrounds, my peers and I filled an important void in a time of need. Our institution was among the first academic medical centers to feel the effects of the COVID-19 crisis, and psychiatry residents played a crucial role in a successful mitigation strategy. My coverage occurred during a time many weeks before the realities of a vast pandemic became apparent. As such, fear and doubt were far from my mind. As COVID-19 continues to test the stability of our healthcare systems, these emotions will be omnipresent. As they were early in the crisis, solidarity, resilience, and flexibility will remain of paramount importance to cope with fear and doubt. I remain encouraged by teamwork that psychiatry and our residency peers demonstrated and will continue to demonstrate.

\section{Gregory Alberto's Reflections}

Away on vacation, I was unaware of the epidemic smoldering in our world's community. By the time I returned, I was all too aware that unusual things were happening. The first grumblings emerged that people were being quarantined, removed 
from their posts to stay home. The next rumor was that this new virus had crept into our hospital's residency programs. The next information I got was not rumor, but reassignment: all to a crescendoing background of media reports regarding the COVID-19 global pandemic.

I arrived directly to a medicine unit that was running lean. Handoff was given from grateful residents who had, diligently, prepared for smooth transition to a single intern. With just a skeleton crew, the workroom felt lonely, but moments of solidarity sparked during brief hallway exchanges with fellow residents. At the end of each day, handoff was given to grateful, even rested, medicine residents and commitment to our shared mission was silently reaffirmed. That first week was marked by pervasive uncertainty. Despite our solidarity, doubt and fear dominated every interaction in the days when initial cases were surfacing and testing was scarce. Who got screened? Tested? What kind of precautions are needed? Who got PPE? Will I get sick? These problems were being worked out in offices behind closed doors, and unclear answers were communicated via e-mail. Ultimately, weak administrative efforts to assuage deep, existential fear were not what carried us. On the floors we marched forward together, necessarily grounded in the shared moment, persevering with humor, food, and the common calling to serve.

It seems only a matter of time before the human resources available during the first week of "lean" operations will be perceived as ample. Doubt and fear will only magnify. It continues to be the call to serve that carries me through uncertainty or fear because, I feel, it is our great privilege as physicians to hold the knowledge of how to intervene upon illness, promote wellness, and pacify the fears of the unwell and dying. I am honored to be surrounded by colleagues in every specialty as we stand together to face this global threat.

\section{Patrick Ho's Reflections}

As a fifth-year resident completing both psychiatry and preventive medicine residencies, only $20 \%$ of my time was spent on clinical duties. My remaining time was dedicated to public health work, legislative advocacy, and health systems research. Balancing these roles provided a unique lens as the threat of COVID-19 magnified. As our psychiatry interns got pulled to cover IM, reports of similar situations began to trickle in from around the country. Simultaneously, I took part in coordinating our state's COVID-19 response as part of my preventive medicine training. While my perspective was unique, I was not insulated from the ennui of uncertainty that my colleagues were feeling. As a preventive medicine resident, when would I be pulled back to cover psychiatry? IM? How would I balance the increased demands of governmental public health work in response to crisis? What did my colleagues and loved ones need, and how could I support them? Was my healthcare work going to jeopardize my spouse's health?

During this crisis, situational awareness seemed elusive due to the circumstance's fluidity. This constant uncertainty certainly made my anxiety harder to ignore. This adversity, though, seems to have galvanized my residency colleagues. The recognition that many of us share anxieties about the uncertain nature of the crisis seemed to pull us together. Seeing colleagues adapt so quickly to uncertainty while continuing to go above and beyond to support each other inspired hope. Although we might find ourselves in unfamiliar (and frankly terrifying) situations, we will do what we can to support each other and our patients.

\section{The Role of Residents in Providing Care during a Crisis}

As residents, we are at the forefront of our communities' efforts to continue providing quality patient care during this crisis. The harsh realities of providing healthcare in the time of COVID-19 will mean that all residents will be in uncharted territories that may be out of each resident's comfort zone, and fear will be an understandable reaction. This early account occurred in a rural setting, but medical centers all over the country have since faced similar challenges - often on an even larger scale as seen in more urban settings. The willingness of psychiatry residents to assist colleagues in times of crisis will ensure that we can continue to care for our patients - even when resources are depleted.

Acknowledgments The authors would like to acknowledge Dr. Christine Finn for her review of a draft of this manuscript.

\section{Compliance with Ethical Standards}

Conflict of interest Drs. Alberto, Rosen, and Ho report no conflicts of interest related to the subject of this article and no proprietary or commercial interest in any product mentioned or concept discussed in this article.

Publisher's Note Springer Nature remains neutral with regard to jurisdictional claims in published maps and institutional affiliations. 